\title{
Effect of social media in improving knowledge among patients having fixed appliance orthodontic treatment: A single-centre randomized controlled trial
}

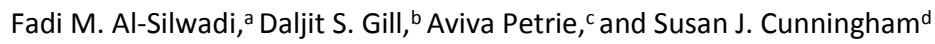

London, United Kingdom

a Honorary specialist registrar, Department of Orthodontics, University College London Eastman Dental Institute.

${ }^{b}$ Consultant orthodontist, Department of Orthodontics, Eastman Dental Hospital, University College London Hospitals NHS Foundation Trust.

c Unit head, Department of Biostatistics, University College London Eastman Dental Institute.

d Professor, Department of Orthodontics, University College London Eastman Dental Institute.

Address correspondence to:

Fadi M. Al-Silwadi, Department of Orthodontics, UCL Eastman Dental Institute, 256 Gray's Inn Rd, London WC1X 8LD, United Kingdom; e-mail, fsilwadi@gmail.com. 


\section{$\underline{\text { ABSTRACT }}$}

Introduction: Research has shown that orthodontic patients are more likely to retain information presented in an audiovisual format. However, there has been little research on the effectiveness of audiovisual information provided through different routes such as the Internet. This parallel-group randomized controlled trial assessed whether provision of audiovisual information on the YouTube (Google, San Bruno, Calif) Web site to orthodontic patients undergoing fixed appliance treatment results in improved patient knowledge when compared with conventional methods of information provision. The effects of sex and ethnicity were also investigated.

Methods: Participants were recruited from the Department of Orthodontics of the Eastman Dental Hospital, University College London Hospitals NHS Foundation Trust, United Kingdom. The patients were 13 years of age and over, with no history of orthodontic treatment, and patient and parental (where appropriate) consent were obtained. The participants were randomized into control $(n=34)$ and intervention $(n=33)$ groups using a random number table; there was stratification based on age group, with permuted blocks of 10 patients. Both groups were given routine verbal and written patient information related to fixed appliances, and the participants in the intervention group were sent 3 e-mails over 6 weeks requesting that they view a 6-minute YouTube video containing similar information but in audiovisual format. Patient knowledge was measured using identical questionnaires answered on the day of recruitment (baseline) and again 6 to 8 weeks later. The researchers were unaware of group allocations when enrolling patients and scoring questionnaires.

Results: Sixty participants (89.55\%) completed the study. Those who completed the trial in the intervention group $(n=30)$ demonstrated significantly greater improvements in knowledge than did those in the control group ( $\mathrm{n} 530$ ), scoring, on average, almost 1 point more $(95 \% \mathrm{Cl}$ for the difference, 0.305-1.602; $\mathrm{P}=0.005$ ) on the final questionnaire. Ethnicity had a statistically significant effect on improvement in knowledge, but sex did not. No harm to any patient was noted during the trial.

Conclusions: Presenting audiovisual information through the YouTube Web site to orthodontic patients resulted in a significant improvement in patient knowledge. Supplementation of verbal and written patient information with audiovisual information via the Internet is therefore worthy of consideration.

Registration: National Research and Ethics Service (REC number 12/LO/0863), United Kingdom.

Protocol: The protocol was not published before trial commencement.

Funding: No funding or conflict of interest to be declared. 
An important goal of health care professionals is to effectively communicate information to patients to improve knowledge. Many authors have examined the role that information provision plays in orthodontic treatment. Information can be transmitted to patients in many ways, and researchers have compared some of them in terms of their effectiveness. Common trends in the way that patients prefer to receive information have been identified.

Patient compliance and effective communication are integral parts of orthodontic treatment, and patients who are compliant during treatment may achieve more favourable treatment outcomes. ${ }^{1-6}$ The communication of certain elements of orthodontic treatment, such as oral hygiene, has been shown to be effective not only during treatment but also in the long term. ${ }^{1}$ Dentists should consider how they communicate instructions to patients to improve their compliance. ${ }^{2}$ Specifically, during orthodontic treatment, it has been shown that patient cooperation increases as communication between the orthodontist and the patient improves. ${ }^{3}$

Patient cooperation depends on many factors, including the establishment of an effective rapport with the treating clinician. ${ }^{4}$ Enhanced communication has been shown to increase patient satisfaction, improve patient knowledge, and provide the motivation required to achieve compliance during treatment. ${ }^{5}$ The methods that orthodontists use to communicate with patients are a vital part of the information provision process because patients must understand what is required of them if they are to be compliant and motivated during treatment. ${ }^{6,7}$ Previous research has shown that providing information to orthodontic patients in a visual format is effective in terms of information retention. ${ }^{8-11}$ In recent years, it has become relevant to study how effective the Internet and social media can be in improving patient knowledge during treatment. In the United States, $32 \%$ of the surveyed consumers stated that they had used social media for health care purposes, but recent studies have shown that hospitals in the United States are not using social media to its full capacity to communicate with patients. ${ }^{12-14}$ There has been little research on the effectiveness of online information in improving patient knowledge; however, it has been shown that orthodontic patients use the Internet for treatment-related information. ${ }^{15}$

Specific objectives or hypotheses

The motivation for undertaking this study was therefore to investigate the potential benefits of using the Internet to provide audiovisual information to orthodontic patients for knowledge improvement. The aim was to determine whether presenting audiovisual information through YouTube (Google, San Bruno, Calif) to orthodontic patients undergoing fixed appliance treatment results in improvements in the knowledge related to appropriate care of the teeth and appliances during treatment.

The null hypothesis for this study was that presenting additional audiovisual information through YouTube to orthodontic patients having fixed appliance treatment does not result in an improvement in knowledge related to appropriate care of the dentition and the appliances compared with conventional provision of information. 
MATERIAL AND METHODS

$\underline{\text { Trial design }}$

This study was designed as a prospective parallel group randomized controlled trial with a 1:1 allocation ratio in which eligible patients who had just had fixed orthodontic appliances placed were randomly allocated into either an intervention or a control group.

Participants, eligibility criteria, and setting

The patients in this trial were all attending the Eastman Dental Hospital, University College London Hospitals NHS Foundation Trust, London, United Kingdom, for fixed appliance orthodontic treatment. The participants included patients of both sexes, aged 13 years and over. Patients were excluded if they did not have a valid e-mail address, had a history of previous orthodontic treatment, were receiving orthodontic treatment with only removable orthodontic appliances or headgear, required complex multidisciplinary treatment, or had a craniofacial syndrome. Ethical approval was obtained from the National Research and Ethics Service (reference number 12/LO/0863), and the University College London Hospitals NHS Foundation Trust Research and Development Department granted research and development approval.

$\underline{\text { Interventions }}$

Patients in the intervention group were given routine verbal and written information; in addition, they were sent 3 e-mails over a period of 6 weeks, at fixed biweekly intervals. The e-mails asked them to view a YouTube video designed specifically for this study containing information relevant to care of their dentition and fixed appliances during treatment (Fig 1). The video was uploaded to the University College London Hospitals YouTube channel as an unlisted video that was accessible only through the Web address provided in the e-mails to the patients in the intervention group. Patients in the control group received the same verbal and written information but did not receive any e-mails directing them to the YouTube video. All patients were asked to answer a 15 -item single-best-answer questionnaire on the day of bond up (baseline data) and an identical questionnaire asking the same questions 6 weeks later at the end of the study. The comparison of data from the 2 questionnaires allowed changes in knowledge to be assessed.

Patients allocated to the intervention group received the standard verbal information given to orthodontic patients and the British Orthodontic Society's information leaflets that are routinely provided to all patients in the department. The video they were asked to watch was developed for this study and provided the same information as in the verbal and written formats, but this time in an audiovisual format. The video centred on 5 major themes: coping with a newly fitted fixed appliance, eating and drinking with a fixed appliance, oral hygiene and its importance, retention, and appliance breakages and emergency appointments.

The 3 biweekly e-mails were identical and asked the patients to follow a link to the YouTube site. The first e-mail was sent on the day that these patients had their fixed appliances fitted and completed the initial questionnaire. The last e-mail was sent 4 weeks later, and the final questionnaire was completed approximately 2 weeks after that, at the first archwire change visit.

The control group did not receive e-mails directing them to view the YouTube video but were still given standard verbal information and the British Orthodontic Society's information leaflets. Therefore, patients allocated to the control group received everything that those in the intervention group did, with 
the exception of the YouTube video. The information provided to the control group participants in both verbal and written forms served 2 major purposes. First, it would be unethical not to provide the usual information; second, this allowed a comparison to be made between the 2 groups relating to the effectiveness of the medium used to transmit information rather than the effectiveness of the information itself.

Outcomes (primary and secondary) and any changes after trial commencement

Two questionnaires were given to all patients in the trial. One was given on the day that the fixed appliances were placed and provided baseline data (Q1), and the second was distributed approximately 6 to 8 weeks later at the patient's first review visit (Q2). The second questionnaire marked the end of the trial for each participant. The 2 questionnaires were identical, although the final questionnaire had 1 additional question, which asked all patients (regardless of their group) whether they had watched the YouTube video. This question accounted for the fact that someone in the intervention group might not have watched the video and the possibility that someone in the control group might have obtained the link and accessed the video. The YouTube video was classified as "unlisted" and could be accessed only through a link that was e-mailed to patients in the intervention group, so this was unlikely. The questionnaire was piloted and amendments made until the final version was developed. Each questionnaire contained 15 single-best answer questions and asked questions closely related to the information presented to both groups of patients.

The outcome measure in this trial was the difference in scores between the 2 questionnaires. The 2 questionnaires for each patient in this study were scored manually by the first author after completion of the second questionnaire, but without knowing the patient's group. Each questionnaire had a total score of 15 , with 1 point for each correct answer. Multiple-choice questions with a single best answer were considered to be an objective assessment, where assessment bias was minimal. All questionnaires were then double marked by a second researcher (D.S.G.) to ensure reliability.

Sample size calculation

It was difficult to establish a standardized difference for a sample size calculation. Therefore, it was decided that an internal pilot would be undertaken to establish the standardized difference and calculate the sample size. This used data from the first 10 patients in each group who completed the trial. A difference in score of 1 point was considered to be a clinically relevant difference between the 2 questionnaires because there were only 15 questions. The standardized difference was then calculated by dividing the clinically relevant difference by the standard deviation of the differences; this was estimated at 1.10 from the pilot study, giving a standardized difference of 0.91 . Based on a paired t test with a $5 \%$ level of significance, a power of $80 \%$, and a standardized difference of 0.91 , the optimal sample size was estimated at 20 patients in each group. However, this was inflated to 30 in each group to account for possible confounding factors and potential dropouts.

$\underline{\text { Interim analyses and stopping rules }}$

It had been planned that an intention-to-treat analysis would be used if patients in the intervention group had not watched the YouTube video or in the unlikely event that a patient in the control group had seen the video. However, this was not required because only 1 patient in the intervention group 
stated that he or she had not watched the video and declined to complete the final questionnaire. No patients in the control group had watched the video.

$\underline{\text { Randomization }}$

To ensure that the groups had similar numbers of patients as the trial progressed, block randomization was used. Randomization was by random number tables and occurred in permuted blocks of 10 patients, so that once 10 patients were recruited, there would be 5 in each group. Patients were stratified into those aged 13 to 15 years and those 16 years and over; a separate randomization list was used in each stratum to ensure that the groups had equal numbers of adults and children as the trial progressed. This was considered important to ensure that confounding bias related to different intelligence levels between the age groups would be minimized. For ethical considerations and to ensure that the patients looked for e-mails if necessary, they were clearly informed which group they were allocated to.

To reduce bias, the researchers were blinded to group allocation when each patient consented; each new patient's group was concealed by placing this information in an opaque envelope that was opened only when the patient consented to be included.

Blinding

The researchers remained blinded to group allocations when scoring the questionnaires and entering the data into the results spreadsheet.

\section{$\underline{\text { Statistical analysis }}$}

The statistical software used to analyze the data in this trial was SPSS (version 22.0; IBM, Armonk, NY).

Baseline demographic data were summarized for the 2 groups. The outcome data collected from the questionnaires (ie, the patient's knowledge level) was continuous, and multivariable regression analysis was used to determine the independent effects of group, sex, ethnicity, and the Q1 questionnaire score on the patient's knowledge in the final questionnaire (Q2). The significance level was set at 0.05 for all hypothesis tests.

\section{RESULTS}

Participant flow

The participants were recruited between November 2012 and February 2014. A CONSORT flow diagram (Fig 2) shows their progression in the study.

Of the 67 patients recruited, 60 completed both questionnaires. Seven patients were lost to follow-up; 6 did not complete the Q2 within the required time frame because of missed appointments, and 1 refused to complete the Q2, stating that he or she had not watched the YouTube video.

\section{Baseline data}

The baseline demographics for the patients who completed the questionnaire are shown in Table I. 
Numbers analyzed for each outcome

All requirements to undertake multivariable regression analysis were satisfied, as judged by a study of the residuals, and the results of the questionnaires and the subsequent analyses are shown in Tables II and III. The dependent variable was the score achieved on the second questionnaire.

This analysis indicated that the patient's allocation group significantly affected the score achieved in the final questionnaire after accounting for the score in the first questionnaire, sex, and ethnicity. Patients in the intervention group scored, on average, almost 1 point higher on the second questionnaire than did those in the control group $(95 \% \mathrm{Cl}$ for the difference, 0.305-1.602; $\mathrm{P}=0.005)$. Sex was not significant, but ethnicity was; patients who were not white achieved higher scores on the Q2 compared with white patients by, on average, 0.798 points ( $95 \% \mathrm{Cl}$ for the difference, $0.158-1.438 ; P=0.016$ ). A screenshot of the YouTube video shows that by February 28, 2014, the site had been viewed 98 times.

The final video was viewed 8 times by the research team before commencing the trial; this means that it was viewed 90 times by participants in the intervention group. This indicates that each of the 30 participants in the intervention group may have watched the video 3 times on average.

$\underline{\text { Harms }}$

No harm was noted during the trial.

\section{DISCUSSION}

Main findings in the context of the existing evidence, Interpretation

The final outcome of orthodontic treatment is the product of many factors. Clinician factors clearly play a part, but patient factors, such as patient knowledge and compliance, are increasingly being recognized as fundamental parts of achieving optimum treatment outcomes. This study showed that giving orthodontic patients audiovisual information through the Internet significantly improved their knowledge. Both groups had improvements in their mean questionnaire scores; this agrees with previous research showing that providing patient information improves their knowledge. ${ }^{9,10}$

Patients in the intervention group scored, on average, almost 1 point higher on the second questionnaire than did those in the control group. This represents approximately a $6 \%$ increased level of knowledge compared with the control group. This observed difference, although statistically significant, was relatively small, and the question of whether it is clinically relevant remains, although it is arguable that any improvement in knowledge is relevant.

The observed difference in knowledge may be large enough to positively impact the outcome of orthodontic treatment because certain aspects of patient compliance such as oral hygiene are vital for all stages of treatment. A meaningful gain in knowledge cannot be accurately assessed by magnitude alone; the area in which knowledge improved is important when determining its impact on the end result.

This study showed that the use of a video transmitted to patients via the Internet is effective in enhancing knowledge. Even greater enhancements of knowledge might have been seen if the video had been produced by those in the video editing profession, and if more resources had been devoted to its production. 
The impact of increased patient knowledge is potentially far reaching, but there is little evidence for this, so definitive conclusions cannot be made. The duration of treatment may be shorter with fewer risks, and there may also be improved treatment outcomes. Moreover, patients may benefit from improved knowledge beyond the end of orthodontic treatment because practices based on that knowledge, such as maintaining optimum oral hygiene, could be retained. Future studies should assess the impact of information provision on patient compliance during treatment (ie, basic periodontal examination score, oral hygiene index score, appliance breakage) and end of treatment outcomes (ie, occlusal outcome scores). It is also important to establish which aspects of patient knowledge are the most significant in contributing to favourable treatment outcomes.

On the second questionnaire, the intervention group patients were allowed to provide comments about the online informational video. The comments were generally positive and indicated that sharing information with patients in an online video can be extremely useful. The patients found the information useful and thought that it should be made more widely available. The provision of information through online social media is a cost-effective method of improving patient knowledge and does not appear to be disadvantageous in any way. Research has also shown that patients show a preference for this type of information. ${ }^{16}$ The positive comments from participants about the online informational video may be explained by the fact that the information was structured and did not focus on marketing any products, and the patients were guided as to where to find the information.

Strengths and limitations

This study, with its strengths and limitations, was conducted as a prospective randomized controlled trial. The YouTube video was intended only for the intervention group patients and was published on the Internet as an unlisted video. The only way that patients in the control group could have gained access to this video was if the link was shared by patients in the intervention group, and this was highly unlikely. The control of access to the intervention during the trial was one of its strengths.

Stratifying for age at recruitment reduced the confounding bias that age could have introduced, particularly due to different intelligence levels. Sex and ethnicity were not stratified for because the conventional randomization process should have dealt with such differences, and it is not normal to stratify for such variables in randomized controlled trials.

The investigators in this study were blinded to which group the participants were recruited to when they consented and during the scoring of the questionnaires. The use of single-best-answer questionnaires provided an objective assessment, which in turn reduced the risk of assessment bias. The relatively short questionnaire (15 questions) led to $100 \%$ completion by those who completed the study; however, the small number of questions, although focusing on the most important aspects of patient knowledge, had some limitations. The small number of questions meant that the difference in scores between Q1 and Q2 might not have completely reflected the difference in knowledge because there was a limit to the number of topic areas covered. A more accurate measure of the difference in knowledge could have been determined if the questionnaires included more questions, but this must be weighed against a decreased response with a longer questionnaire.

The overall female:male ratio of $66.7 \%: 33.3 \%$ reflects the orthodontic population, in which female patients predominate. ${ }^{17}$ The majority of the patients in the study were white $(60 \%)$, reflecting the 
demographics in the United Kingdom. The ratios of white to not white were similar in the 2 groups; this is important in terms of bias, especially when considering that ethnicity was shown to significantly affect the Q2 score. The equal numbers in the 2 age groups were made possible by the randomization process.

Generalisability

The generalisability of the observed results may be limited because this trial was undertaken at 1 centre, and relatively short questionnaires were used.

\section{CONCLUSIONS}

The null hypothesis in the study was rejected. It appears that presenting audiovisual information through the YouTube site to orthodontic patients undergoing fixed appliance orthodontic treatment does, on average, result in an improvement in knowledge related to care of the dentition and the appliances when compared with standard methods of providing information.

\section{REFERENCES}

1. Zachrisson BU. Oral hygiene for orthodontic patients: current concepts and practical advice. Am J Orthod 1974;66:487-97.

2. Bartsch $A$, Witt $E$, Marks $M$. The influence of information and communication in the orthodontic consultation and treatment visit. II. The communication style and compliance. Fortschr Kieferorthop 1995;56:7-15.

3. Nanda RS, Kierl MJ. Prediction of cooperation in orthodontic treatment. Am J Orthod Dentofacial Orthop 1992;102:15-21.

4. Sinha PK, Nanda RS, McNeil DW. Perceived orthodontist behaviors that predict patient satisfaction, orthodontist-patient relationship, and patient adherence in orthodontic treatment. Am J Orthod Dentofacial Orthop 1996;110:370-7.

5. Mehra T, Nanda RS, Sinha PK. Orthodontists' assessment and management of patient compliance. Angle Orthod 1998;68:115-22.

6. Sinha PK, Nanda RS. Improving patient compliance in orthodontic practice. Semin Orthod 2000;6:237-41.

7. Brattstro€m V, Ingelsson M, Aberg E. Treatment co-operation in orthodontic patients. Br J Orthod 1991;18:37-42.

8. Thomson AM, Cunningham SJ, Hunt NP. A comparison of information retention at an initial orthodontic consultation. EurJ Orthod 2001;23:169-78.

9. Anderson MA, Freer TJ. An orthodontic information package designed to increase patient awareness. Aust Orthod J 2005;21:11-8.

10. Patel JH, Moles DR, Cunningham SJ. Factors affecting information retention in orthodontic patients. Am J Orthod Dentofacial Orthop 2008;133(Suppl):S61-7.

11. Srai J, Petrie A, Ryan F, Cunningham SJ. The effect of information provision on anxiety in adolescent orthodontic patients. Am J Orthod Dentofacial Orthop 2013;144:505-11.

12. Pricewaterhouse Coopers (PwC) Health Research Institute. Socialmedia "likes" healthcare: From marketing to social business. Available at: http://www.pwc.com/us/en/health-industries/publications/ health-care-social-media.jhtml. Accessed March 28, 2012.

13. Thaker SI, Nowacki AS, Mehta NB, Edwards AR. How U.S. hospitals use social media. Ann Intern Med 2011;154:707-8.

14. Verasoni Worldwide. Hospitals \& facebook: a case study, 2011. Available at: http://www.verasoni.com/ahha3/2216/. Accessed May 1, 2012.

15. Kn€osel M, Jung K. Informational value and bias of videos related to orthodontics screened on a video-sharing Web site. Angle Orthod 2011;81:532-9.

16. Livas C, Delli K, Ren Y. Quality evaluation of the available Internet information regarding pain during orthodontic treatment. Angle Orthod 2013;83:500-6.

17. Harris EF, Glassell BE. Sex differences in the uptake of orthodontic services among adolescents in the United States. Am J Orthod Dentofacial Orthop 2011;140:543-9. 


\section{FIGURES}

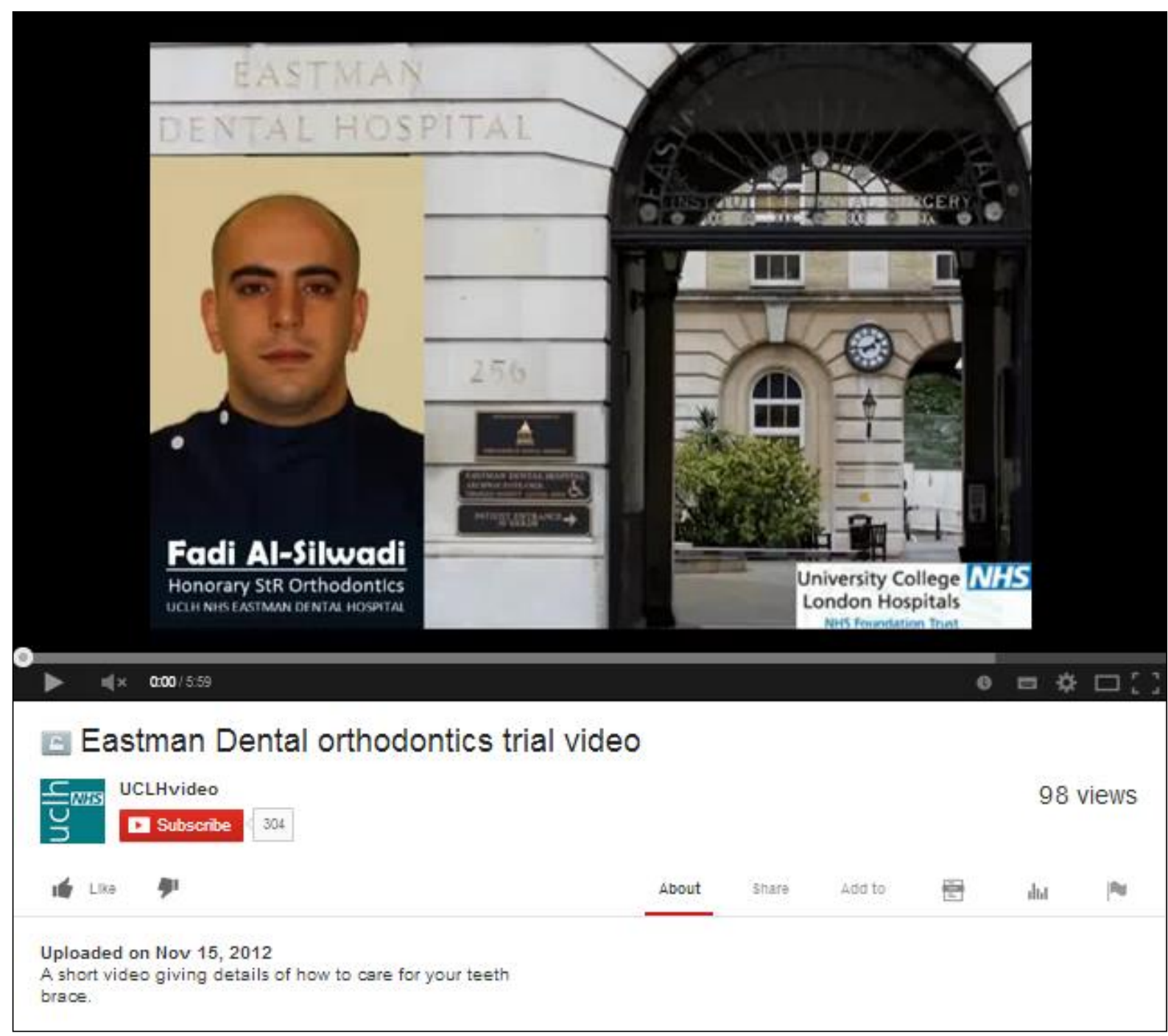

Figure I. YouTube ${ }^{\mathrm{TM}}$ Webpage containing the unlisted video. 


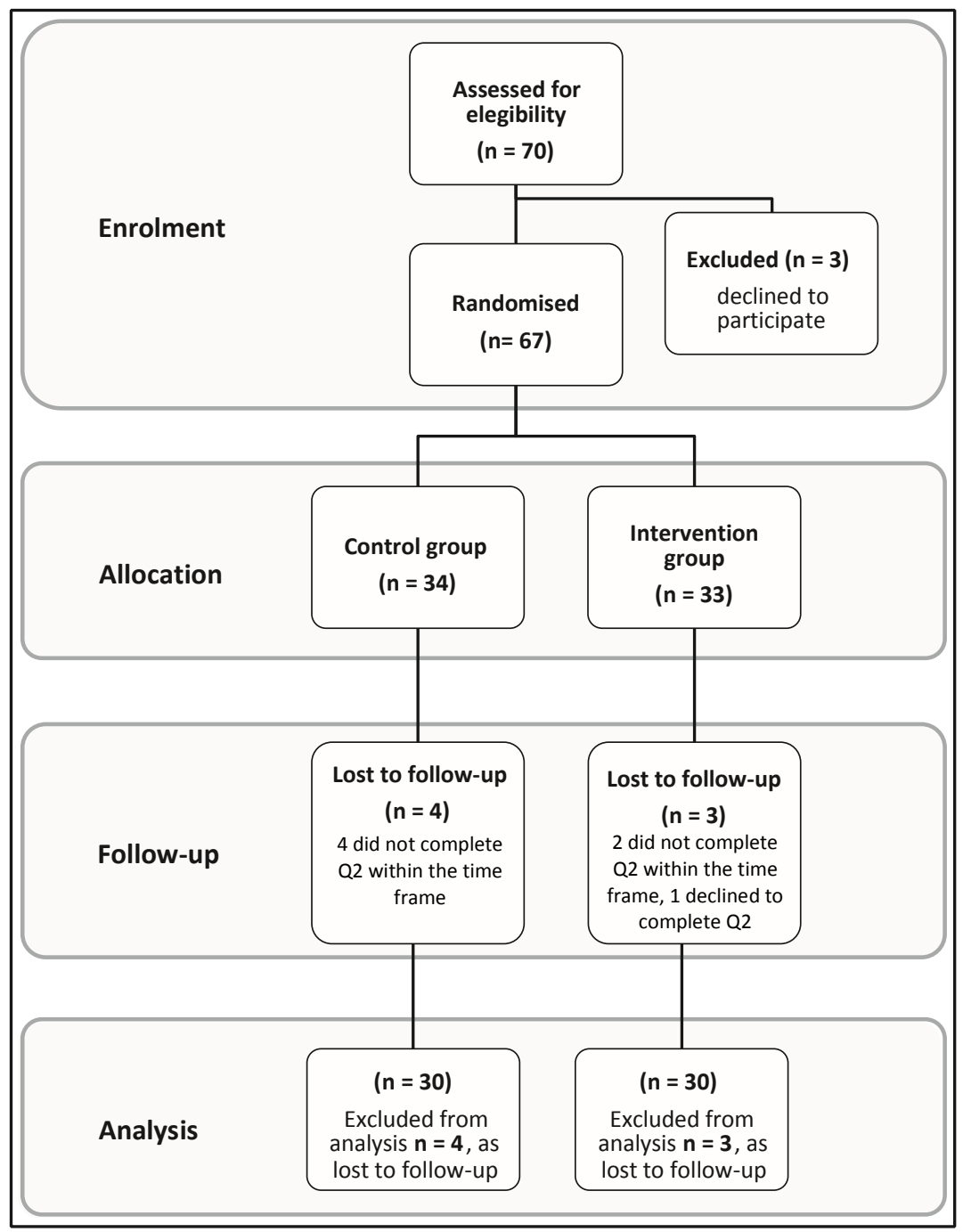

Figure II. CONSORT flow diagram of participants in the study. 
$\underline{\text { TABLES }}$

\begin{tabular}{|c|c|c|c|c|}
\hline & & & & \\
\hline Demogra & & Control & Intervention & Total \\
\hline Gender & Male & $5 \quad(16.7 \%)$ & 15 (50\%) & $20(33.3 \%)$ \\
\hline [n (\%)] & Female & 25 (83.3\%) & 15 (50\%) & 40 (66.7\%) \\
\hline Fth & Caucasian & $16(53.3 \%)$ & 20 (66.7\%) & 36 (60\%) \\
\hline & $\begin{array}{l}\text { Non- } \\
\text { Caucasian }\end{array}$ & $14(46.7 \%)$ & 10 (33.3\%) & 24 (40\%) \\
\hline Median a & years] & 15.50 & 15.50 & 15.50 \\
\hline
\end{tabular}

Table I. Baseline demographics of the patients who completed both questionnaires.

\begin{tabular}{|l|c|c|c|c|c|c|}
\cline { 2 - 7 } \multicolumn{1}{c|}{} & \multicolumn{3}{c|}{ Control Group (n=30) } & \multicolumn{2}{c|}{ Intervention Group (n=30) } \\
\cline { 2 - 7 } \multicolumn{1}{c|}{} & Mean & SD & Median & Mean & SD & Median \\
\hline $\begin{array}{l}\text { Initial } \\
\text { Questionnaire }\end{array}$ & 12.63 & 1.47 & 13.00 & 12.77 & 1.61 & 13.00 \\
(Q1) & 12.70 & 1.51 & 13.00 & 13.67 & 1.21 & 14.00 \\
\hline Final & & & & & & \\
(Q2) & 0.07 & 1.66 & 0.00 & 0.90 & 1.27 & 1.00 \\
\hline Difference & & & & & & \\
\hline (Q2-Q1) & & & & & & \\
\hline
\end{tabular}

Table II. Scores and difference in scores between initial and final questionnaires for both groups.

Predictors: Ethnicity, Gender, Q1, Group

Dependent Variable: Q2 


\begin{tabular}{|c|c|c|c|c|c|}
\hline \multirow{2}{*}{ Model } & \multicolumn{2}{|c|}{$\begin{array}{l}\text { Unstandardized } \\
\text { Coefficients }\end{array}$} & \multicolumn{2}{|c|}{ 95.0\% Confidence Interval } & \multirow{2}{*}{ P-value } \\
\hline & B & $\begin{array}{l}\text { Std. } \\
\text { Error }\end{array}$ & Lower Bound & $\begin{array}{l}\text { Upper } \\
\text { Bound }\end{array}$ & \\
\hline (Constant) & 6.694 & 1.284 & 3.636 & 10.506 & $<0.001$ \\
\hline $\begin{array}{l}\text { Group } \\
0 \text { - Control } \\
1 \text { - Intervention }\end{array}$ & 0.953 & 0.324 & 0.305 & 1.602 & 0.005 \\
\hline Q1 Score & 0.507 & 0.102 & 0.302 & 0.712 & $<0.001$ \\
\hline $\begin{array}{l}\text { Gender } \\
0 \text { - Female } \\
1 \text { - Male }\end{array}$ & 0.157 & 0.341 & -0.526 & 0.840 & 0.648 \\
\hline $\begin{array}{l}\text { Ethnicity } \\
0 \text { - Non-Caucasian } \\
1 \text { - Caucasian }\end{array}$ & -0.798 & 0.319 & -1.438 & -0.158 & 0.016 \\
\hline
\end{tabular}

Table III. Multivariable regression analysis incorporating group, score on the initial questionnaire (Q1), gender, and ethnicity 